\title{
MULTIPLIERS ON COMPLEX HOMOGENEOUS SPACES
}

\author{
R. C. GUNNING ${ }^{1}$
}

1. Let $G$ be a real Lie group, represented as a transitive group of analytic automorphisms of a simply-connected complex analytic manifold $D$; if $g \in G$ and $z \in D$, the action of the transformation representing $g$ on the point $z$ will be denoted by $g z$. A multiplier for the group $G$, with respect to its representation as a transformation group on $D$, is a $C^{\infty}$ complex-valued function $\mu(g ; z)$ on $G \times D$ which is holomorphic in $z$ and which satisfies $\mu\left(g_{1} g_{2} ; z\right)=\mu\left(g_{1} ; g_{2} z\right) \mu\left(g_{2} ; z\right)$ for every $g_{1}, g_{2} \in G$; to exclude the obvious trivial case, we further assume that $\mu(g ; z) \not \equiv 0$. Such functions are sometimes considered in examining the group $G$ and its representations, ${ }^{2}$ but also arise as the continuous analogs of some structures of interest in the study of automorphic functions $;^{3}$ our purpose here is to determine the possible multipliers which may arise in connection with the second of the above points of view. We shall always assume here that $G$ is connected. Notice that the set of all multipliers for $G$ forms an abelian group $\mathfrak{T}(G ; D)$ under multiplication.

The universal covering group $G^{*}$ of $G$ also acts as a transformation group on $D$, the action of the transformation representing $g^{*} \in G^{*}$ on the point $z \in D$ being defined by $g^{*} z=g z$ whenever $g^{*}$ covers $g$; we shall consider firstly the group $\operatorname{Tr}\left(G^{*} ; D\right)$ of multipliers for $G^{*}$. Let $H^{*}$ be the isotropy subgroup of $G^{*}$ at some point $z_{0}$, which point is to be held fixed subsequently, and let $K^{*}$ be the subgroup of $G^{*}$ consisting of all elements represented by the trivial transformation which leaves $D$ pointwise fixed. For our purposes, in particular for Siegel's modular groups, there is no loss of generality in assuming: (i) that there are local $C^{\infty}$ mappings $z \rightarrow g_{z}^{*}$ of $D$ into $G^{*}$ such that $g_{3}^{*} z_{0}$ $=z$; (ii) that $K^{*}$ is the center of $G^{*}$; (iii) that $K^{*} \cap\left[G^{*}, G^{*}\right]=e^{*}$, that is, the intersection of the center and the commutator subgroup of $G^{*}$ is the trivial subgroup consisting of the identity $e^{*}$ alone; and (iv) that elements of finite order are everywhere dense in the group $H^{*} / K^{*}$.

Whenever $f(z)$ is holomorphic and nowhere vanishing on $D$,

Received by the editors April 27, 1956.

1 This note was prepared while the author was a National Science Foundation post-doctoral fellow.

${ }^{2}$ V. Bargmann, Irreducible unitary representations of the Lorentz group, Ann. of Math. vol. 48 (1947).

${ }^{8}$ R. C. Gunning, The structure of factors of automorphy, Amer. J. Math. vol. 78 (1956). 
$\mu_{0}\left(g^{*} ; z\right)=f\left(g^{*} z\right) f(z)^{-1}$ is a multiplier; these are called the trivial multipliers, and form a subgroup $\mathrm{J}\left(G^{*} ; D\right)$ of $\mathfrak{N}\left(G^{*} ; D\right)$ which is canonically isomorphic to the group of holomorphic, nowhere-vanishing functions on $D$. Let $\mathcal{L}\left(G^{*} ; D\right)$ be the additive group of $G^{*}$-invariant differential forms of type $(0,1)$ on the manifold $D$ which are of the form $\bar{\partial} g(z, \bar{z})$ for some $C^{\infty}$ complex valued function $g(z, \bar{z})$ on $D$; in the cases which arise from automorphic functions, when $D$ is a Stein manifold, these differential forms are just the $\bar{\partial}$-closed $G^{*}$ invariant forms of type $(0,1)$. Finally let $\operatorname{Hom}\left(K^{*} ; C\right)$ be the group of $C^{\infty}$ homomorphisms of $K^{*}$ into the additive group of complex numbers.

TheOREM. The group $\mathfrak{T}\left(G^{*} ; D\right)$ is canonically isomorphic to a direct sum as follows:

$$
\mathfrak{T}\left(G^{*} ; D\right) \cong \mathscr{L}\left(G^{*} ; D\right) \oplus \Im\left(G^{*} ; D\right) \oplus \operatorname{Hom}\left(K^{*} ; C\right) .
$$

Proof. Since $G^{*} \times D$ is simply-connected, $\sigma\left(g^{*} ; z\right)=\log \mu\left(g^{*} ; z\right)$ is a well-defined single-valued function, that branch of the logarithm being selected for which $\sigma\left(e^{*} ; z\right)=0$ for the identity $e^{*} \in G^{*}$; moreover $\sigma\left(g_{1}^{*} g_{2}{ }^{*} ; z\right)=\sigma\left(g_{1}{ }^{*} ; g_{2}{ }^{*} z\right)+\sigma\left(g_{2}{ }^{*} ; z\right)$ for every $g_{1}{ }^{*}, g_{2}{ }^{*} \in G^{*}$. In particular, whenever $k^{*} \in K^{*}$ and $g^{*} \in G^{*}$, it follows from assumption (ii) that $g^{*-1} k^{*} g^{*}=k^{*}$, and hence that $\sigma\left(k^{*} ; z\right)=\sigma\left(g^{*-1} k^{*} g^{*} ; z\right)=\sigma\left(g^{*-1}\right.$; $\left.k^{*} g^{*} z\right)+\sigma\left(k^{*} ; g^{*} z\right)+\sigma\left(g^{*} ; z\right)=\sigma\left(k^{*} ; g^{*} z\right)$; therefore $\sigma\left(k^{*} ; z\right)=\hat{\sigma}\left(k^{*}\right)$ is a constant. The mapping $k^{*} \rightarrow \hat{\sigma}\left(k^{*}\right)$ is an element of $\operatorname{Hom}\left(K^{*} ; C\right)$, and the mapping $\mu\left(g^{*} ; z\right) \rightarrow \hat{\sigma}$ is a homomorphism of $\operatorname{Tr}\left(G^{*} ; D\right)$ into Hom $\left(K^{*} ; C\right)$. Now restricting ourselves to the kernel of the above homomorphism in $\Re\left(G^{*} ; D\right)$, we have $\sigma\left(k^{*} ; z\right)=0$ for every $k^{*} \in K^{*}$. Whenever $h^{*} \in H^{*}$ corresponds to an element of finite order in $H^{*} / K^{*}$, say $h^{*^{n}} \in K^{*}$, then $0=\sigma\left(h^{*^{n}} ; z_{0}\right)=n \sigma\left(h^{*} ; z_{0}\right)$; but since such elements are everywhere dense in $H^{*}$ by assumption (iv), it follows that $\sigma\left(h^{*} ; z_{0}\right)=0$ for every $h^{*} \in H^{*}$. Thus for every $g^{*} \in G^{*}, g_{0 * z}^{*} z_{0}$ $=g^{*} z=g^{*} g_{z}^{*} z_{0}$, so that $g_{0 * z}^{*-1} g^{*} g_{z}^{*} \in H^{*}$; consequently $0=\sigma\left(g_{0 * z}^{*-1} g^{*} g_{z}^{*} ; z_{0}\right)$ $=-\sigma\left(g_{0 * z}^{*} ; z_{0}\right)+\sigma\left(g^{*} g_{z}^{*} ; z_{0}\right)$. Now $f(z)=\sigma\left(g_{z}^{*} ; z_{0}\right)$ is independent of the choice of local sections $g_{2}^{*}$, is clearly a $C^{\infty}$ function on $D$ by assumption (i), and for any $g^{*} \in G^{*}, f\left(g^{*} z\right)=\sigma\left(g_{0 * 2}^{*} ; z_{0}\right)=\sigma\left(g^{*} g_{z}^{*} ; z_{0}\right)$ $=\sigma\left(g^{*} ; z\right)+f(z)$. Obviously any other function satisfying this functional equation differs from $f(z)$ at most by an additive constant. To each $\sigma\left(g^{*} ; z\right)$ associate the differential form $\bar{\partial} f(z)$; this defines a homomorphism of the set of multipliers with $\hat{\sigma}=0$ into the group $\mathcal{L}\left(G^{*} ; D\right)$, and the kernel is clearly precisely the group $J\left(G^{*} ; D\right)$. To complete the proof, we need merely show that the above homomorphisms are onto. For any differential form $\bar{\partial} f(z) \in \mathcal{L}\left(G^{*} ; D\right)$, 
$\mu(g ; z)=\exp (f(g z)-f(z))$ is a multiplier having $\hat{\sigma}=0$ and mapping onto the form $\bar{\partial} f(z)$ by the previous mapping. Further, any element $\hat{\sigma} \in \operatorname{Hom}\left(K^{*} ; C\right)$ can be extended to an element $\hat{\sigma}_{1} \in \operatorname{Hom}\left(G^{*} ; C\right)$; for if we map $G^{*}$ homomorphically onto the abelianized group $G^{*} /\left[G^{*}, G^{*}\right]$, this will be an isomorphism on $K^{*}$ by assumption (iii), and since a homomorphism on a subgroup of an abelian group can be extended to the full group, $\hat{\sigma}$ clearly admits the desired extension, the exponential of which is a multiplier mapping onto the element $\hat{\sigma}$. This therefore concludes the proof.

Any homomorphic image $G_{1}$ of $G^{*}$ for which the kernel $K_{1}^{*}$ of the homomorphism $G^{*} \rightarrow G_{1}$ is contained in $K^{*}$ likewise acts as a transformation group on $D$; the multipliers $\mathfrak{\Re}\left(G_{1} ; D\right)$ are determined by the subgroup of $\operatorname{Tr}\left(G^{*} ; D\right)$ consisting of those multipliers $\mu\left(g^{*} ; z\right)$ for which $\mu\left(k_{1}^{*} ; z\right)=1$ whenever $k_{1}^{*} \in K_{1}^{*}$.

2. As an example, consider the symplectic group acting on the generalized unit disc of degree $p$, as introduced by Siegel. ${ }^{4}$ The assumptions as listed previously are fulfilled in this case. Moreover the group $\mathscr{L}\left(G^{*} ; D\right)$ is a one-dimensional vector space over the complex numbers. To see this, recall that there is but one independent, closed $G$-invariant differential form of type $(1,1)$ on $D$ in this case, namely the form $\Omega$ determined by the metric. ${ }^{4}$ If $\theta_{1}, \cdots, \theta_{a}$ form a basis for $\mathcal{L}\left(G^{*} ; D\right)$, the elements $\partial \theta_{1}, \cdots, \partial \theta_{a}$ must be dependent; hence by a suitable choice of the base, we may assume that $\partial \theta_{2}=\cdots=\partial \theta_{a}=0$. But then $\theta_{j} \wedge \bar{\theta}_{j}$ will be a closed and invariant form for $j \geq 2$, and must be zero since $\Omega$ does not admit such a decomposition ${ }^{5}$ hence $\theta_{j}=0$ for $j \geq 2$, and $a=1$. Therefore in this case, the nonobvious multipliers in $\mathfrak{T}\left(G^{*} ; D\right)$ are powers of the Jacobian determinants of the transformations representing elements of $G^{*}$. The same is of course true whenever there is but one closed invariant form of type $(1,1)$ on $D$.

\section{University of Chicago}

4. L. Siegel, Symplectic geometry, Amer. J. Math. vol. 65 (1943).

- This would imply $\Omega \wedge \Omega=0$, which is impossible for a Kaehler metric. 